\section{AESA AG / Allgemeine Einkaufszentrale der Schweizer Ärzte}

\author{
Information der FMH an ihre Mitglieder \\ François-X. Deschenaux, Generalsekretär FMH \\ Erika Flückiger, Leiterin Dienste FMH
}

Vor sechs Jahren haben die FMH und die Ärztekasse zusammen mit der CMCG Genf die Allgemeine Einkaufszentrale der Schweizer Ärzte AESA gegründet mit dem Zweck, im Markt von medizinischem Verbrauchsmaterial und medizinischen Geräten in der Schweiz mehr Konkurrenz zu schaffen und damit der schweizerischen Ärzteschaft eine echte Alternative zu bieten. Mit ihrem Erscheinen und Agieren im Schweizer Markt hat die AESA eines ihrer Hauptziele, die Senkung der Preise für diese Güter, erreicht: die Preise sind in den letzten Jahren generell um 10-15\% gesunken. Davon hat die ganze Ärzteschaft, nicht nur die Kunden der AESA, profitiert.

Leider ist es nicht gelungen, eine genügend grosse Anzahl Ärztinnen und Ärzte für die Idee einer Einkaufszentrale zu gewinnen. Die AESA hat deshalb die kritische Grösse nicht erreichen können und musste Ende Juni 2001 ihre Geschäftstätigkeit einstellen. Aus betrieblicher Sicht hat die AESA Schiffbruch erlitten.
Der Geist, der ihrer Gründung zu Gevatter stand, ist aber geblieben und soll auch in Zukunft bewahrt bleiben. Um unseren Mitgliedern die Dienstleistung, welche bisher die AESA erbracht hat, zu erhalten und die Unannehmlichkeiten, die mit der Aufgabe der Geschäftstätigkeit dieser Firma unweigerlich einhergehen, so gering wie nur möglich zu halten, haben wir uns nach einem starken Partner umgesehen.

In der Galexis AG, der für den Geschäftsbereich Distribution zuständigen Firma der Galenica Gruppe, haben wir diesen Partner gefunden. Dank des Einsatzes der Galexis, die über mordernste Verteilzentren in Bern-Schönbühl, Lausanne-Ecublens und ZürichSchlieren verfügt, konnte die Erledigung der bei der AESA noch pendenten Bestellungen und ausstehenden Teillieferungen inzwischen sichergestellt werden.

Wie oben erwähnt, wollen wir die strategische Zielsetzung, die wir mit unserer Beteiligung an der AESA verfolgten, unter etwas anderen Rahmenbedingungen weiterführen. Wir stehen deshalb mit der Galexis AG auch in Verhandlungen über eine langfristige Lösung, über die wir Sie zu gegebener Zeit wieder informieren werden. Selbstverständlich steht Ihnen die Galexis jetzt schon für die Entgegennahme Ihrer Bestellungen über die folgenden Telefon- und Faxnummern zur Verfügung:

- Kundendienst Bern-Schönbühl, Grubenstrasse 11, 3322 Schönbühl, Tel. 03185874 00, Fax 031 85874 10;

- Kundendienst Zürich-Schlieren, Zürcherstrasse 13, 8952 Schlieren, Tel. 0173394 30, Fax 01 73394 33;

- Kundendienst Lausanne-Ecublens, Route de Crochy 2, 1024 Ecublens, tél. 02169453 02, fax 0216945333. 